\title{
Interactions Among Small Interfering RNAs And MicroRNAs in Arabidopsis Thaliana
}

\author{
Pontes, O., Vitins, A., Pikaard, C.S., Hong, E., and Ream, T. \\ Biology Department, Washington University, 1 Brookings Drive, St. Louis, MO 63130, USA.
}

In organisms as diverse as humans, flies, nematodes, fission yeast and plants, miRNAs (micro RNAs) and/or siRNAs (small interfering RNAs) regulate numerous essential processes. These include inactivation of mRNAs in development, viral defense, silencing of transposons/endogenous repeats and proper centromere functioning, as required for accurate chromosome segregation to daughter cells in each round of cell division $[1,2,3,4]$. In general, siRNAs or miRNAs that are generated by Dicer (DCL) endonucleases are stably associated with Argonaute (AGO) proteins within RNA-induced silencing complexes (RISCs). RISCs can direct RNA-dependent DNA methylation and heterochromatin formation at homologous genes to cause transcriptional silencing or they can mediate translational arrest or cleavage of homologous mRNAs to silence genes posttranscriptionally.

Arabidopsis thaliana has an elaborate set of proteins involved in small RNA biogenesis. Many of these proteins function in distinct pathways but there is substantial crosstalk and functional redundancy among components of the pathways. Critical for small RNA production are the four DCL proteins that act in partnership with various additional activities. For instance, DCL1 is primarily responsible for miRNA production while DCL3 is involved in the biogenesis of heterochromatic siRNA that target DNA methylation. These Dicers also contribute to siRNA production associated with transgene silencing or viral defense, along with DCL2 and DCL4 [5]. There is clear collaboration between the DCL1-mediated production of miRNAs and the resulting production of tasiRNAs, which involves DCL4 dicing of precursors that are generated by miRNAdirected cleavage and subsequent RNA-DEPENDENT RNA POLYMERASE 6 (RDR6)-dependent production of double-stranded RNA (dsRNA) [5]. Until recently, it was thought that the DCL3dependent pathway for heterochromatic siRNA production, involving Nuclear RNA polymerase IV (pol IV), RDR2 and AGO4 was devoted solely to transcriptional gene silencing and RNA-dependent DNA methylation (RdDM) [6]. However, Pol IV and RDR6, but not RDR2, are also required for production of regulatory siRNAs generated by DCL2 from dsRNAs formed where the 3 ' ends of adjacent gene transcription units overlap [5].

Our previous cytological analysis of siRNA-directed DNA methylation pathway strongly suggests a stepwise model for nuclear siRNA biogenesis and target locus chromatin modifications in a process that involves a hypothetical siRNA-processing center (Figure1A) [7] similar to Cajal-Bodies (Figure1B) [8] and the periphery of heterochromatic regions. On the other hand, an analogous nucleolar structure for DCL1 has been described, designated as dicing bodies [9], in which all the proteins required for primary miRNA transcript processing are colocalizing with pri-miRNA precursors, suggesting that these nuclear bodies represent miRNA processing centers. Similarly to the DCL3 processing centers we described, DCL1 processing centers/dicing bodies are associated with the nucleolus but is not clear how they are related to Cajal bodies and/or DCL3 processing centers.

To address the relationship between Arabidopsis small RNA processing centers and Cajal-body like structures we performed a cell biology based approach to the main components involved in small 
RNA biogenesis. Our data show physical associations among proteins within the nucleus that might help explain not only the partial functional redundancies among the four dicers, but also the functional interactions occurring among small RNA pathway components, resulting in various transcriptional or post-transcriptional gene silencing outcomes.

[2] Baulcombe, D. (2004). RNA silencing in plants. Nature 431, 356-363.

[3] Hannon, G.J., Rivas, F.V., Murchison, E.P., and Steitz, J.A. (2006). The expanding universe of noncoding RNAs. Cold Spring Harbor symposia on quantitative biology 71, 551-564.

[4] Lippman, Z., and Martienssen, R. (2004). The role of RNA interference in heterochromatic silencing. Nature 431, 364-370.

[5] Brodersen, P., and Voinnet, O. (2006). The diversity of RNA silencing pathways in plants. Trends Genet 22, 268-280.

[6] Matzke, M., Kanno, T., Huettel, B., Daxinger, L., and Matzke, A.J. (2006). RNA-directed DNA methylation and Pol IVb in Arabidopsis. Cold Spring Harbor symposia on quantitative biology 71, 449-459. [7]Pontes, O., Li, C.F., Nunes, P.C., Haag, J., Ream, T., Vitins, A., Jacobsen, S.E., and Pikaard, C.S. (2006). The Arabidopsis chromatin-modifying nuclear siRNA pathway involves a nucleolar RNA processing center. Cell 126,79-92.

[8]Li, C.F., Pontes, O., El-Shami, M., Henderson, I.R., Bernatavichute, Y.V., Chan, S.W., Lagrange, T., Pikaard, C.S., and Jacobsen, S.E. (2006). An ARGONAUTE4-containing nuclear processing center colocalized with Cajal bodies in Arabidopsis thaliana. Cell 126, 93-106.

[9]Fang, Y., and Spector, D.L. (2007). Identification of nuclear dicing bodies containing proteins for microRNAs biogenesis in living Arabidopsis plants. Curr Biol 17, 818-823.

A

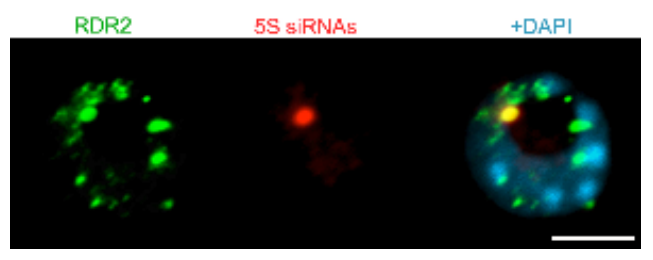

B

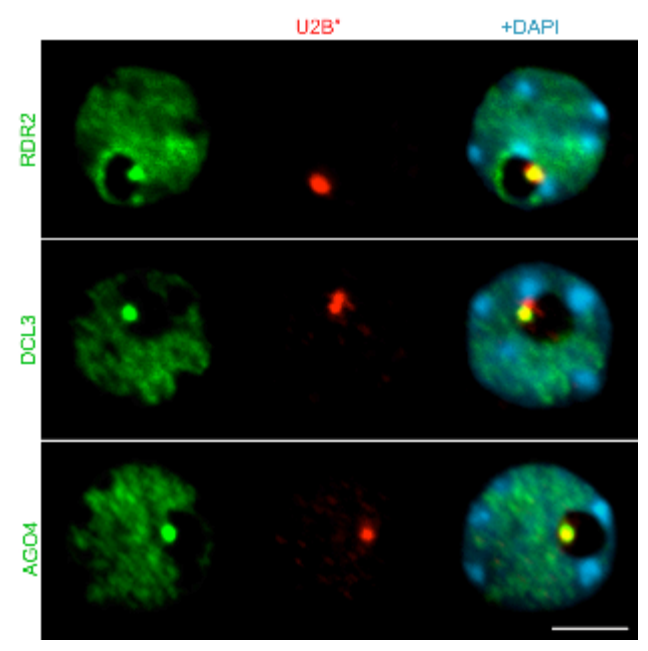

Figure 1. siRNA nuclear localization and relation with Cajal-Body structures. RDR2, DCl3, AGO4 and NRPD1b are colocalized in U2B"-containing Cajal Bodies. A. Heterochromatic siRNAs colocalize with RdDM proteins within the nucleolus in a potential siRNA-processing center. RNA-FISH using a probe corresponding the 5S rRNA gene (5S siRNA) followed by immunolocalization of RDR2 using a native antibody. B. U2B" specific antibody (red) was immunolocalized in lines expressing RDR2-YFP, DCL3-Flag, AGO4-cMyc and NRPD1b-Flag proteins, detected by its respective epitope tags (green). In merged images a strong yellow-labeled dot is shown, resulting from the overlapping green and red fluorescent channels, indicating that the proteins are colocalized. DNA was stained with DAPI and the size bar indicates $5 \mu \mathrm{m}$. 